\title{
Magnetic Properties of Disordered Heisenberg Binary System with long-range exchange
}

\author{
G. X. Tang 1,2 , 因 and W. Nolting ${ }^{1}$ \\ ${ }^{1}$ Institut für Physik, Humboldt-Universität zu Berlin, Newtonstraße 15, D-12489 Berlin, Germany \\ ${ }^{2}$ Department of Physics, Harbin Institute of Technology, D-150001 Harbin, People's Republic of China
}

(Dated: September 25, 2018)

\begin{abstract}
The influence of substitutional disorder on the magnetic properties of disordered Heisenberg binary spin systems with long-range exchange integrals is studied. The equation of motion for the magnon Green's function which is decoupled by the Tyablikov approximation is solved in the BlackmanEsterling-Berk(BEB) coherent potential approximation(CPA) framework, where the environmental disorder term is treated by virtual crystal approximation. The long-range exchange integrals include a power-law decaying and an oscillating Ruderman-Kittel-Kasuya-Yosida(RKKY) exchange interaction. The resulting spectral density, which is calculated by CPA self-consistent equation, is then used to estimate the magnetization and Curie temperature. The results show, in the case of the three-dimensional simple cubic systems, a strong influence of ferromagnetic long-range exchange integrals on the magnetization and Curie temperature of the systems, which is obviously different from the calculation of short-range interaction.
\end{abstract}

PACS numbers: 75.10.Jm, 75.25.+z, 75.30Et

\section{INTRODUCTION}

The effect of disorder has been shown to be crucial for many essential properties of magnetic materials. However, investigations involving statistical disorder are always faced with lots of technical difficulties, especially in a parameter regime that is not accessible to perturbation theory ${ }^{1}$. The introduction and application of the coherent potential approximation(CPA), which is initially developed by Soven ${ }^{2}$ and Taylor ${ }^{3}$, has increased greatly our understanding of disordered systems, particularly of random, substitutional disordered alloys. In the CPA, one assumes that the real, disordered material is replaced by a translationally invariant effective medium. This is fixed by imposing the self-consistent condition that the scattering off of a real atom embedded in the medium vanishes on the average. The CPA provides reliable results in various situations studying excitations like electrons,

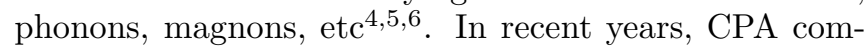
bined with some $a b$ initio method has been shown to be crucial tool for the understanding of diluted magnetic semiconductors(DMS) ${ }^{7.8}$.

For spin systems with substitutional disorder, the problem more generally considered is a binary system ${ }^{9}$ in which the magnetic atoms of each component (A or B) with spin $S_{A}$ or $S_{B}$ are randomly distributed over the $N$ sites of a regular lattice with concentrations $c_{A}$ and $c_{B}\left(c_{A}+c_{B}=1\right)$, respectively. In the present work, the interaction between spins is described by the Heisenbergtype Hamiltonian in which exchange constants are $J_{i j}^{\lambda \lambda^{\prime}}$ : $\lambda, \lambda^{\prime}=A$ or $B$ according to the two interacting spins at site $i$ and $j$. Using Tyablikov decoupling approximation to the retarded magnon Green's function of a Heisenbergtype Hamiltonian, we can achieve the equation of motion for Green's function involving three kinds of disorder that depend explicitly on site spin (diagonal disorder), the exchange parameters (off-diagonal disorder) and an envi- ronmental term given by the static field induced at one site by the presence of neighboring spins (environmental disorder). We will deal with diagonal disorder and off-diagonal disorder terms in the BEB-CPA framework. The BEB theory by Blackman, Esterling and Berk ${ }^{10}$ extends the CPA which studies systems only with diagonal disorder to random off diagonal matrix elements of the Hamiltonian (off-diagonal disorder) by doubling the Hilbert space (for a binary alloy) and adding the atomic sort to the matrix indices 11.12 . The environmental disorder term is treated in the virtual crystal approximation in the same manner as done by Theumann ${ }^{9}$ (to be referred to as I), where an atom $\mathrm{A}(\mathrm{B})$ will be acted upon by a mean field so that full randomness will be preserved in the locator expression of CPA. Furthermore, considering the Tyablikov decoupling approximation for the disordered Green's function, magnetization of the different constituents need to be determined self-consistently for a given temperature because the locator and the local magnon spectral density are temperature dependent. In general, the analytical form of the local magnon spectral density can be got only for some simple cases such as the exchange integrals restricted to only nearest neighbors 13 . Analytically, the calculations where $J_{i j}$ are long ranged is not an easy task. However, long-range interaction are always of interest in different fields of physics because they can give rise to a variety of unusual macroscopic behavior. For example, metallic magnetic materials are of itinerant type, which means that the exchange integrals between different localized magnetic ions are long range and driven by the polarization of the conduction carrier gas, i.e. the Ruderman-Kittel-Kasuya-Yosida (RKKY) type exchange interaction ${ }^{14}$.

In the present work, we calculate magnetization and Curie temperature of different components with ferromagnetic short-range, power-law decaying long-range and oscillating RKKY long-range exchange interaction. 
It should be mentioned here that the idea of using the virtual crystal approximation and BEB-CPA to deal with three kinds of disorder(diagonal, off-diagonal and environmental) of a binary spin system is same as paper I. However, our approach to solve the self-consistent CPA equations, which differs from paper I, follows the BEBCPA method. Moreover, we present the method to calculate the temperature dependence of magnetization and the Curie temperature of systems by combining the methods of paper I and the RPA-CPA theory ${ }^{13}$ done by G. Bouzerar et. al. Significantly, we can calculate any range exchange integrals(including short-range and longrange).

The article is organized as follows: In section III we deal with different types of disorder and derive the configurational averaged Green's functions, magnetization and Curie temperature for systems with ferromagnetic shortrange, ferromagnetic long-range and RKKY-type longrange exchange interactions. The numerical calculations of the self-consistent equations are discussed in III for exchange integrals of a long-range power-law decay $\left|J_{R}\right| \sim$ $R^{-\alpha}$ and a RKKY-type $\left|J_{R}\right| \sim(R \cos R-\sin R) / R^{4}$. In section [V] we will conclude the article with a summary.

\section{THE MODEL}

To study the magnetic properties of a material $\mathrm{A}_{c_{A}} \mathrm{~B}_{c_{B}}$ carrying a localized magnetic moment at each lattice site, we assume the dynamics of these spins to be reasonably described by the isotropic Heisenberg Hamiltonian

$$
H=-\sum_{i, j} J_{i j} \mathbf{S}_{i} \cdot \mathbf{S}_{j}-\frac{1}{\hbar} g_{J} \mu_{\mathrm{B}} B \sum_{i} S_{i}^{z},
$$

where subscripts $i$ and $j$ refer to the occupied lattice sites, and $\mathbf{S}_{i}=\left(S_{i}^{x}, S_{i}^{y}, S_{i}^{z}\right)$ is the spin operator of the localized magnetic moment at lattice site $i$ with lattice vector $\mathbf{R}_{i}$. The Hamiltonian contains a Zeeman coupling of the spins, where the external magnetic field is $\mathbf{B}=$ $(0,0, B)$. The effective Heisenberg exchange parameters $J_{i j}$ obey $J_{i j}=J\left(\left|\mathbf{R}_{i}-\mathbf{R}_{j}\right|\right)$ and $J_{i i}=0$ restricting the theory to uniform media and disregarding more general case. Firstly, we generalize the Hamiltonian (1) such that $J_{i j}$ is random:

$$
\begin{aligned}
J_{i j} & =J_{i j}^{A A} & & \text { if sites } i, j \text { are of type A } \\
& =J_{i j}^{B B} & & \text { if they are of type B } \\
& =J_{i j}^{A B}=J_{i j}^{B A} & & \text { if one is A site and the other B. }
\end{aligned}
$$

Now introduce occupation indices $x_{i}$ and $y_{i}$, such that $x_{i}=1, y_{i}=0$ if $i$ is an A site, $x_{i}=0, y_{i}=1$ if $i$ is an B site.

Some examples of their properties are:

$$
\begin{aligned}
& x_{i} y_{i}=0, \quad x_{i}^{2}=x_{i}, \\
& \left\langle x_{i}\right\rangle_{c}=c_{A}, \quad\left\langle y_{i}\right\rangle_{c}=c_{B},
\end{aligned}
$$

where the bracket $\langle\ldots\rangle_{C}$ means a configurational average. Then, we get

$$
J_{i j}=x_{i} J_{i j}^{A A} x_{j}+x_{i} J_{i j}^{A B} y_{j}+y_{i} J_{i j}^{B A} x_{j}+y_{i} J_{i j}^{B B} y_{j}
$$

Introducing the retarded magnon Green's function

$$
G_{i j}(E)=\left\langle\left\langle S_{i}^{+} ; S_{j}^{-}\right\rangle\right\rangle_{E}^{r e t}
$$

built up by the step operators $S_{i}^{ \pm}=S_{i}^{x} \pm i S_{i}^{y}$, its equation of motion reads

$$
\begin{gathered}
\left(E-g_{J} \mu_{\mathrm{B}} B\right) G_{i j}(E)=2 \hbar^{2} \delta_{i j}\left\langle S_{i}^{z}\right\rangle-2 \hbar \sum_{m} J_{i m} \\
\times\left(\left\langle\left\langle S_{i}^{+} S_{m}^{z} ; S_{j}^{-}\right\rangle\right\rangle_{E}^{r e t}-\left\langle\left\langle S_{m}^{+} S_{i}^{z} ; S_{j}^{-}\right\rangle\right\rangle_{E}^{r e t}\right) .
\end{gathered}
$$

Making the Tyablikov approximation, which consists in decoupling the higher Green's function on the rhs. of (4), one obtains after rearrangement:

$$
\begin{aligned}
{\left[E-g_{J} \mu_{\mathrm{B}} B\right.} & \left.-2 \hbar \sum_{m} J_{i m}\left\langle S_{m}^{z}\right\rangle\right] G_{i j}(E) \\
& =2 \hbar^{2}\left\langle S_{i}^{z}\right\rangle \delta_{i j}-2 \hbar\left\langle S_{i}^{z}\right\rangle \sum_{m} J_{i m} G_{m j}
\end{aligned}
$$

Substituting $J_{i m}$ by equation (2), we use the various combinations $\{x, y\}$ to multiple the equation (5) in BEB manner. Then, the Green's functions' equation of motion can be expressed as,

$$
\mathbb{G}_{i j}=\mathcal{G}_{i}\left(\hbar \delta_{i j}-\sum_{m} \mathbb{J}_{i m} \mathbb{G}_{m j}\right),
$$

if we introduce the $2 \times 2$ matrices

$$
\begin{gathered}
\mathbb{G}_{i j}=\left(\begin{array}{ll}
G_{i j}^{A A} & G_{i j}^{A B} \\
G_{i j}^{B A} & G_{i j}^{B B}
\end{array}\right)=\left(\begin{array}{ll}
x_{i} G_{i j} x_{j} & x_{i} G_{i j} y_{j} \\
y_{i} G_{i j} x_{j} & y_{i} G_{i j} y_{j}
\end{array}\right), \\
\mathbb{J}_{i j}=\left(\begin{array}{ll}
J_{i j}^{A A} & J_{i j}^{A B} \\
J_{i j}^{B A} & J_{i j}^{B B}
\end{array}\right)
\end{gathered}
$$

and

$$
\mathcal{G}_{i}=\left(\begin{array}{cc}
x_{i} g^{A} & 0 \\
0 & y_{i} g^{B}
\end{array}\right)=\left(\begin{array}{cc}
x_{i} \frac{\sigma_{A}}{\omega-\omega_{A}} & 0 \\
0 & y_{i} \frac{\sigma_{B}}{\omega-\omega_{B}}
\end{array}\right)
$$

where $\sigma_{\lambda}=\left\langle S_{\lambda}^{z}\right\rangle / \sigma_{0}\left(\lambda=\mathrm{A}\right.$ or $\mathrm{B}, \sigma_{0}=c_{A}\left\langle S_{A}^{z}\right\rangle+$ $\left.c_{B}\left\langle S_{B}^{z}\right\rangle\right), \omega=\left(E-g_{J} \mu_{\mathrm{B}} B\right) / 2 \hbar \sigma_{0}$ and

$$
\begin{aligned}
\omega_{A} & =\sum_{m}\left(J_{i m}^{A A}\left\langle S_{m}^{z}\right\rangle x_{m}+J_{i m}^{A B}\left\langle S_{m}^{z}\right\rangle y_{m}\right) / \sigma_{0}, \\
\omega_{B} & =\sum_{m}\left(J_{i m}^{B A}\left\langle S_{m}^{z}\right\rangle x_{m}+J_{i m}^{B B}\left\langle S_{m}^{z}\right\rangle y_{m}\right) / \sigma_{0} .
\end{aligned}
$$

Now we find, as in BEB, that the matrix propagator $\mathbb{G}_{i j}$ satisfies an equation for a problem with only local disorder, the matrix $\mathbb{J}_{i j}$ being independent of the random numbers. However, the so called 'local potential' $\omega_{A}$ and $\omega_{B}$ are functions of random variables. Using the virtual crystal approximation as done by paper I, the equation (8) can be written as

$$
\begin{aligned}
& \omega_{A}=c_{A} \sigma_{A}\left(\sum_{m} J_{i m}^{A A}\right)+c_{B} \sigma_{B}\left(\sum_{m} J_{i m}^{A B}\right) \\
& \omega_{B}=c_{A} \sigma_{A}\left(\sum_{m} J_{i m}^{B A}\right)+c_{B} \sigma_{B}\left(\sum_{m} J_{i m}^{B B}\right) .
\end{aligned}
$$


Considering, for any site $i, \sum_{m} J_{i m}^{\lambda \lambda^{\prime}}=$ constant in an exchange-integral and lattice-structure given spin system, the 'local potential' $\omega_{A}$ and $\omega_{B}$, which describe local site occupation, are constants. In other words, the exchange interaction of neighbor spins of site $i$ leads to a static field which acts on site $i$. The static field is of independence on the location of site $i$ as the external magnetic field $\mathbf{B}$ does. Thus, in our approach, the exchange interaction of neighbor spins of site $i$ is taken as an additional static field. The difference between $\omega_{A}$ and $\omega_{B}$ root in whether an atom of type A or of type B occupies site $i$. In our model, the random A-type or B-type occupation of any site decide the 'local potential' $\omega_{A}$ or $\omega_{B}$, respectively. From here, our calculation will follow the BEB-CPA methods and differ from paper I.

If we refer to three specific $\omega$-dependent parameters as a matrix form in BEB-CPA manner

$$
\mathbb{U}_{0}=\left(\begin{array}{ll}
U_{1} & U_{3} \\
U_{3} & U_{2}
\end{array}\right)
$$

the CPA self-consistent condition is expressed as 11

$$
\gamma \equiv\left\langle\mathbb{G}_{i i}\right\rangle_{c}=N^{-1} \sum_{\mathbf{k}} \mathbb{G}_{\mathbf{k}}
$$

where $\mathbb{G}_{\mathbf{k}}=\left[\gamma^{-1}+\mathbb{U}_{0}+\mathbb{J}_{\mathbf{k}}\right]^{-1}$ and

$$
\mathbb{J}_{\mathbf{k}}=\left(\begin{array}{cc}
J^{A A}(\mathbf{k}) & J^{A B}(\mathbf{k}) \\
J^{B A}(\mathbf{k}) & J^{B B}(\mathbf{k})
\end{array}\right)
$$

where $J^{\lambda \lambda^{\prime}}(\mathbf{k})$ is the Fourier transform of the parameters of exchange interaction $J^{\lambda \lambda^{\prime}}(R)$. The renormalized locator can be written as

$$
\gamma=\left(\begin{array}{cc}
\gamma^{A} & 0 \\
0 & \gamma^{B}
\end{array}\right)
$$

where

$$
\begin{aligned}
\gamma^{A} & =\frac{c_{A} \sigma_{A}}{\omega-\omega_{A}-\sigma_{A} U_{1}}, \\
\gamma^{B} & =\frac{c_{B} \sigma_{B}}{\omega-\omega_{B}-\sigma_{B} U_{2}} .
\end{aligned}
$$

In general, the introduced $2 \times 2$ matrix $\mathbb{U}_{0}$ describes effective medium properties 9.11 . From the denominator of the renormalized locators (11), we find $\mathbb{U}_{0}$ has the same dimensions of exchange parameters $J$. So, according to BEB-CPA theory, the effective medium parameters $\mathbb{U}_{0}$ present the appropriate sum of the exchange integrals and are called the renormalized interactor.

Once we get two renormalized locators $\gamma^{A}$ and $\gamma^{B}$ from CPA self-consistent equations, the magnon spectral function can be expressed as

$$
D_{\lambda}(\omega)=-\frac{1}{\pi} \frac{\operatorname{Im} \gamma^{\lambda}(\omega)}{c_{\lambda} \sigma_{\lambda}}
$$

Furthermore, magnetization reads

$$
\left\langle S_{\lambda}^{z}\right\rangle=\hbar \frac{\left(S_{\lambda}-\Phi_{\lambda}\right)\left(1+\Phi_{\lambda}\right)^{2 S_{\lambda}+1}+\left(1+S_{\lambda}+\Phi_{\lambda}\right) \Phi_{\lambda}^{2 S_{\lambda}+1}}{\left(1+\Phi_{\lambda}\right)^{2 S_{\lambda}+1}-\Phi_{\lambda}^{2 S_{\lambda}+1}}
$$

where $\lambda=A$ or $B$ and the average magnon number $\Phi_{\lambda}$ can be calculated by

$$
\Phi_{\lambda}=\int_{-\infty}^{+\infty} d \omega \frac{D_{\lambda}(\omega)}{e^{2 \hbar \sigma_{0} \omega / k_{B} T}-1} .
$$

Now, for a given temperature and good starting value of $\left\langle S_{\lambda}^{z}\right\rangle$, we can get the renormalized locators $\gamma$ and the magnon spectral functoin $D_{\lambda}(\omega)$ from CPA selfconsistent equations (10). Furthermore, by using the equation (13), we can calculate new values of magnetization $\left\langle S_{\lambda}^{z}\right\rangle$, which are re-inserted in $\sigma_{\lambda}$ and $\gamma^{\lambda}$.

Considering $\left\langle S_{\lambda}^{z}\right\rangle \rightarrow 0$ in the limit $T \rightarrow T_{C}$, we can get the Curie temperature

$$
k_{B} T_{C}=\frac{2}{3} \hbar^{2} \sum_{\lambda} c_{\lambda} \frac{S_{\lambda}\left(S_{\lambda}+1\right)}{F_{\lambda}}
$$

where

$$
F_{\lambda}=\int_{-\infty}^{+\infty} d \omega \frac{D_{\lambda}(\omega)}{\omega}
$$

To study the influence of the range of a ferromagnetic exchange interaction on magnetization, we need to calculate $J^{\lambda \lambda^{\prime}}(\mathbf{k})$ and $\sum_{m} J_{i m}^{\lambda \lambda^{\prime}}$ with ferromagnetic decaying long-range

$$
J^{\lambda \lambda^{\prime}}(R)= \begin{cases}J_{0}^{\lambda \lambda^{\prime}}(R / a)^{-4} & \text { for } R \leq R_{\text {cut-off }} \\ 0 & \text { otherwise }\end{cases}
$$

Here, $R_{\text {cut-off }}$ is the maximum distance out of which the spin-spin interaction is not taken into account in the calculations, $a$ is the lattice constant and $J_{0}$ the nearestneighbor interaction strength. For $R_{\text {cut-off }}=a$, we get the case of nearest-neighbor interaction $J=J_{0}$, otherwise $J=0$. At the same time, in the numerical studies, we also use RKKY-type long-range exchange interaction

$$
J^{\lambda \lambda^{\prime}}(R)=J_{0}^{\lambda \lambda^{\prime}}\left(\frac{R}{a}\right)^{-4}\left[\sin \left(\frac{R}{a}\right)-\left(\frac{R}{a}\right) \cos \left(\frac{R}{a}\right)\right] .
$$

Considering $J^{\lambda \lambda^{\prime}}(R)$ depending on the shell $R$, we have

$$
\sum_{m} J_{0 m}^{\lambda \lambda^{\prime}}=\sum_{I} z_{I} J^{\lambda \lambda^{\prime}}\left(R_{I}\right)
$$

where $\sum_{I}$ corresponds to a summation over the I-th shell with a distance $R_{I}$ from a given site 0 and $z_{I}$ is the total number of spin in the shell. We also get a shell expression

$$
J^{\lambda \lambda^{\prime}}(\mathbf{k})=\sum_{I} J^{\lambda \lambda^{\prime}}\left(R_{I}\right) \sum_{\mathbf{r}_{m}^{I}} \exp \left(i \mathbf{k r}_{m}^{I}\right)
$$

where the sum $\mathbf{r}_{m}^{I}$ runs over each site located in the I-th shell. 


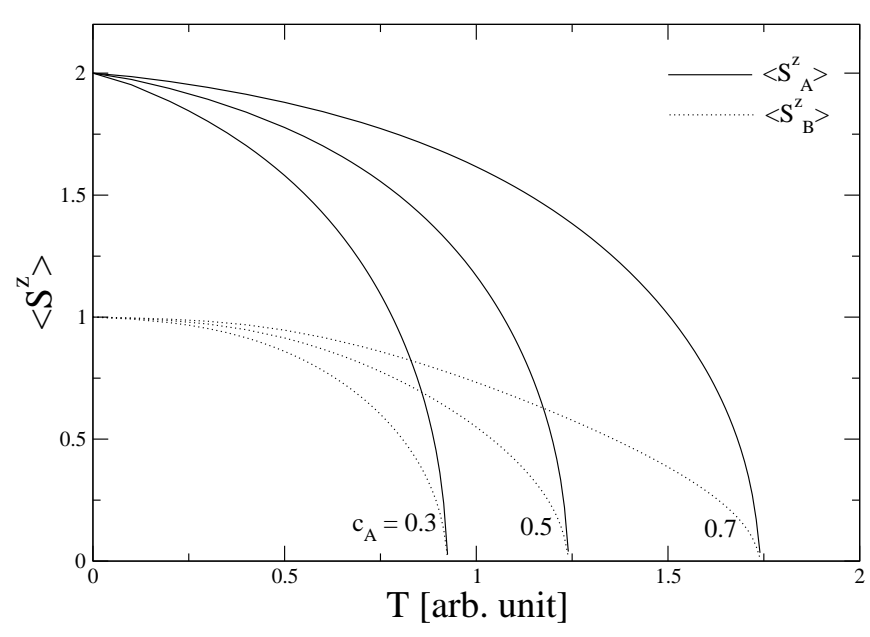

FIG. 1: Magnetization $\left\langle S^{z}\right\rangle$ as function of temperature $T$ for nearest-neighbor interaction $\left(R_{c u t-o f f}=a\right.$ for power-law decaying long-range exchange integrals) on a simple cubic lattice for various concentrations $c_{A}$. The parameters are $J_{0}^{A A}=0.2$, $J_{0}^{A B}=0.1, J_{0}^{B B}=0.15, S_{A}=2$ and $S_{B}=1$.

\section{NUMERICAL STUDIES}

In this section, we will mainly consider the influence of long-range exchange integrals on Curie temperature of a three-dimension disordered binary simple cubic systems with different exchange constants. For simplicity, we only consider the case of a zero external field and the lattice constant equals to 1 .

The magnetization of two different spin components as a function of temperature is shown in Fig. 11 for several different concentrations $c_{A}$. The figure shows that each spin component's magnetization equals to their spin quantum number at $T=0$ and will decrease monotonically with increasing temperature. For any concentration $c_{A},\left\langle S_{A}^{z}\right\rangle$ and $\left\langle S_{B}^{z}\right\rangle$ will decrease to zero at the same temperature. It is because the exchange integrals between two components is ferromagnetic and the ferromagnetic ordering will disappear at the Curie temperature.

In Fig. 2] we show the Curie temperature as a function of the maximum length $R_{\text {cut-off }}$ of exchange interaction, i.e. the spin-spin interaction will not be taken into account out of $R_{\text {cut-off }}$. For power-law decaying long-range exchange integrals, it can be found in Fig. 2 that the Curie temperature increases monotonically with increasing $R_{\text {cut-off }}$ and has a saturation value at $R_{\text {cut-off }} \rightarrow \infty$ for any concentration. For RKKYtype long-range exchange integrals, the Curie temperature firstly increases with increasing $R_{c u t-o f f}$ and then presents an oscillating behavior around a fixed value, which corresponds to $R_{\text {cut-off }} \rightarrow \infty$. Both calculated results in Fig. 2] show that long-range exchange integrals will lead to changes of the Curie temperature and magnetic properties of spin systems compared to the shortrange case. The results also show that the long range of the interaction has to be taken into account explicitly in a
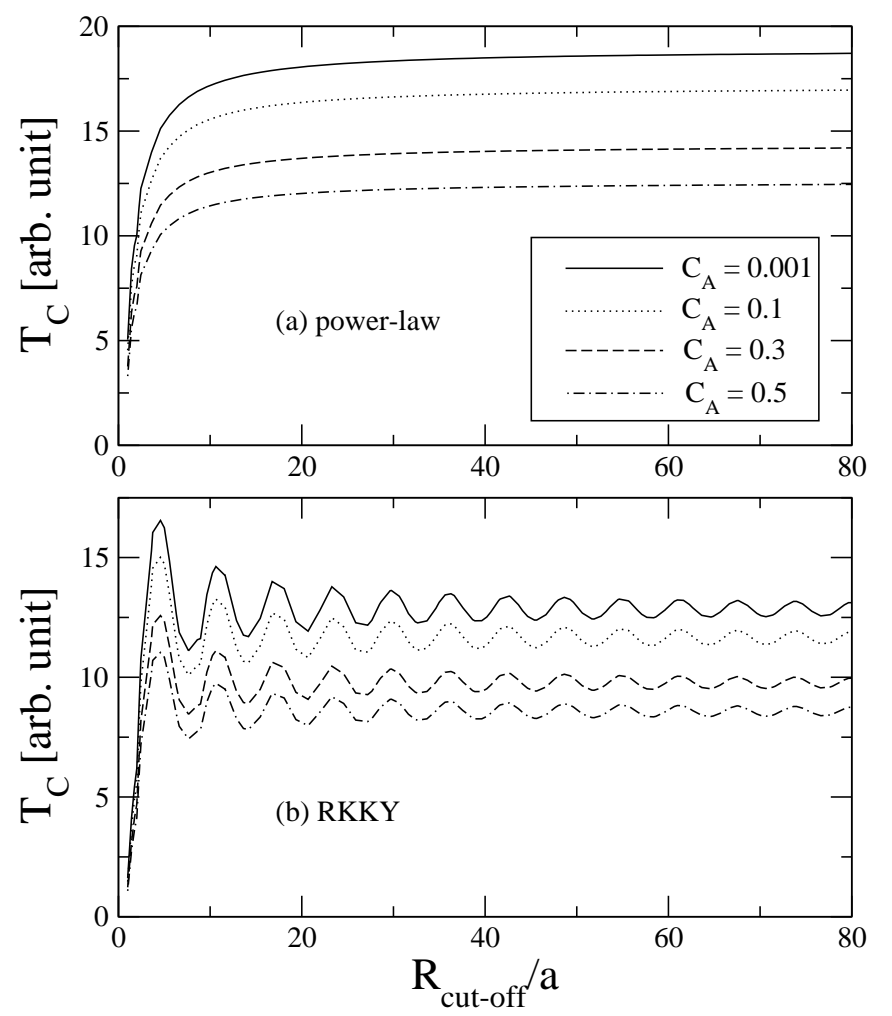

FIG. 2: Curie temperature $T_{\mathrm{C}}$ as function of the effective radius $R_{c u t-o f f} / a$ (a: lattice constant) for (a) power-law decaying and (b) RKKY-type long-range exchange integrals on a sc lattice. The parameters are $J_{0}^{A A}=0.2, J_{0}^{A B}=0.1$, $J_{0}^{B B}=0.15, S_{A}=2$ and $S_{B}=3$.

respective model calculation. To cut at a too early stage, for mathematic simplicity, may lead to rather misleading results.

Furthermore, considering various ratios of $c_{A}$ to $c_{B}$ that affect $T_{\mathrm{C}}$, the Curie temperature as a function of $c_{A}$ for nearest-neighbor and infinite long-range exchange integrals $\left(R_{\text {cut-off }} \rightarrow \infty\right)$ is shown in Fig. 3] At the same time, the figures show the influence of three different strengths $J_{0}^{A B}$ on the Curie temperature. Note that pure $\mathrm{A}$ (or B) corresponds to $c_{A}=1$ (or $\left.c_{A}=0\right)$. Compared with other methods 13 , the Curie temperature in the left column of Fig. 3] which is the result of nearestneighbor exchange parameters, present similar tendency for different values of $J_{0}^{A B}$. The spin $S_{A}, S_{B}$ and exchange integrals $J^{\lambda \lambda^{\prime}}\left(\lambda, \lambda^{\prime}=A\right.$ or $\left.B\right)$ of the binary spin system will influence magnetization and Curie temperature corporately. Comparing the left column to the right column of the Fig. 3] we find that the Curie temperature of long-range exchange integrals is hugely different from the Curie temperature of nearest-neighbor exchange integrals. 

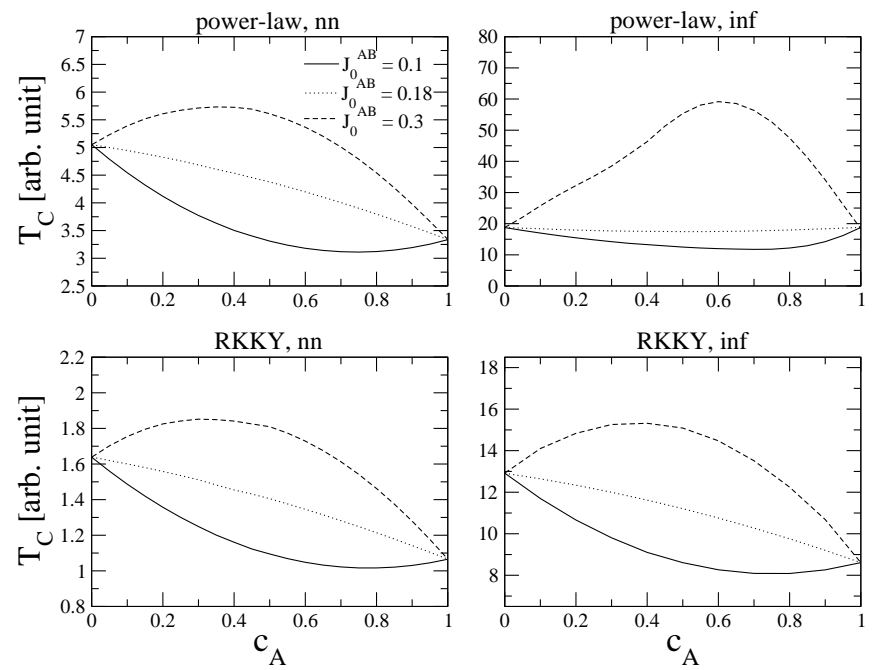

FIG. 3: Curie temperature $T_{\mathrm{C}}$ as function of the concentration $c_{A}$ for power-law decaying and RKKY-type long-range exchange integrals on a sc lattice, where nn means nearestneighbor exchange parameters and inf means $R_{c u t-o f f} \rightarrow \infty$. Note the different scales for $T_{\mathrm{C}}$ at the left and the right column. The parameters are $J_{0}^{A A}=0.2, J_{0}^{B B}=0.15, S_{A}=2$ and $S_{B}=3$.

\section{SUMMARY}

The aim of this article is mainly to study the influence of long-range exchange integrals on the ferromagnetic properties of random spin systems. By mak- ing the Tyablikov approximation to equation of motion for the magnon Green's function, we investigate substitutional random spin systems in BEB-CPA framework. The resulting theory is then solved numerically in a selfconsistent way for a simple cubic systems. The obtained spectral densities are then used to calculate spontaneous magnetization and estimate the Curie temperature of the corresponding system.

For ferromagnetic power-law decaying and RKKYtype long-range exchange interactions, the calculations show long-range exchange integrals will lead to changes of macroscopic properties such as the Curie temperature of spin systems. The Curie temperature will oscillate for RKKY-type long-range exchange interaction and increase monotonically for power-law decaying interaction with the effective range $R_{\text {cut-off }}$ of exchange integrals. It will tend to a saturation value for $R_{\text {cut-off }} \rightarrow \infty$. These results imply that the effective exchange interaction, numerically taken into account, must be very long-ranged. The influence of long-range interaction on magnetization and Curie temperature should carefully be considered in the theoretical model.

\section{Acknowledgments}

We thank Mr. Bryksa for helpful discussions. G.X.T is supported by the State Scholarship Programs of China Scholarship Council, the National Natural Science Foundation of China under Grant No. 50375040 and Foundation of HIT Grant No. HIT.MD2002.16.
* Electronic address: tang@physik.hu-berlin.de

1 R. Vlaming, and D. Vollhardt, Phys. Rev. B, 45, 4637 (1992).

2 P. Soven, Phys. Rev., 156, 809 (1967).

3 D. W. Taylor, Phys. Rev., 156, 1017 (1967).

${ }^{4}$ R. J. Elliott, J. A. Krumhansl, and P. L. Leath, Rev. Mod. Phys., 46, 465(1974).

5 J. P. Julien, P.E.A. Turchi, and D. Mayou, Phys. Rev. B, 64, 195119(2001).

6 D. A. Rowlands, J. B. Staunton, and B. L. Györffy, Phys. Rev. B, 67, 115109(2003).

7 L. Sandratskii, P. Bruno, and J. Kudrnovský, Phys. Rev. B69, 195203(2004).

${ }^{8}$ K. Sato, P. H. Dederichs, and H. Katayama-Yoshida, Europhys. Lett. 61, 403(2003).

9 A. Theumann, J. Phys. C, 7, 2328(1974).

10 J. A. Blackman, D. M. Esterling, and N. F. Berk, Phys. rev. B, 4, 2412(1971).

11 A. Gonis, and J.W. Garland, Phys. Rev. B, 16, 1495(1977).

12 K. Koepernik, B. Velický, R. Hayn, and H. Eschrig, Phys. Rev. B, 58, 58(1998).

${ }^{13}$ G. Bouzerar, and P. Bruno, Phys. Rev. B, 66, 014410(2002).

14 M. A. Ruderman, and C. Kittel, Phys. Rev., 96, 99(1954); T. Kasuya, Prog. Theor. Phys., 16, 45(1956); K. Yosida,
Phys. Rev., 106, 893(1957). 